\title{
Palliative Care and Rehabilitation for Stroke Survivors: Managing Symptoms and Burden, Maximizing Function
}

\author{
Sydney M. Dy, MD, MSC ${ }^{1,3}$ and Dorianne R. Feldman, MD, MSPT ${ }^{2}$ \\ 'Departments of Health Policy and Management, Oncology, and Medicine, Johns Hopkins University, Baltimore, MD, USA; ${ }^{2}$ Physical Medicine \\ and Rehabilitation, Johns Hopkins University, Baltimore, MD, USA; ${ }^{3}$ Baltimore, MD, USA.
}

KEY WORDS: palliative care; rehabilitation; stroke; survivors; cerebrovascular accident; pain.

J Gen Intern Med 27(7):760-2

DOI: $10.1007 / \mathrm{s} 11606-012-2054-0$

(C) Society of General Internal Medicine 2012

$\mathrm{S}$ troke survivors often have permanent disfigurement, dysphonia, dysphagia, or sialhorrhea. Frequently, they are wheelchair bound or have gait deviations, and are limited or dependent on others for activities of daily living and bowel and bladder care. They may have cognitive impairment and/or inability to communicate or comprehend conversations. Commonly, they develop musculoskeletal, neuropathic, and/or other painful syndromes which further impair physical functioning and quality of life.

These individuals may be hospitalized at a stroke center far away from their local residence and then be transferred to other levels of care for months while recuperating. Once discharged, many are left with significant deficits and are now reliant on a caregiver, whether parent, spouse, sibling, or close friend. This can lead to estrangement from social roles and networks for both the patient and caregiver, and often there is no specialty stroke care available once the patient is at home. Caregivers may have a poor understanding of the role which they are undertaking, and are often inadequately trained to perform necessary care. In addition, patients and caregivers may have difficulties with negative emotions and adjustment to their permanently changed status, as well as anxiety and depression.

In this issue of JGIM, Creutzfeldt et al. ${ }^{1}$ describe evidence for symptomatic and palliative care management in stroke survivors, focusing on several specific pain syndromes, a variety of other physical and psychological symptoms, caregiver support, and spiritual needs. Addressing these issues is critical for the primary care physician, and both the palliative care and rehabilitation perspectives provide useful approaches. Recent reviews ${ }^{2}$ have found that the existing literature on palliative care in stroke patients is limited, mostly focusing on acute stroke care and end-of-life issues, such as uncertainty of prognosis, acute symptoms, and decision-making. Studies have found that symptoms (such as pain and dyspnea) are prevalent in acute stroke care

Published online April 13, 2012 as well; that the caregiving burden is profound and longlasting; and that decision-making about issues such as artificial nutrition in severely impaired patients has particular challenges.

Many concepts in cancer palliative care and survivorship are translatable to survivors of stroke, although there are also fundamental differences. As in cancer, survivorship issues are best considered from the time of diagnosis, so that the long-term sequelae of life-sustaining treatments and manageable chronic disease, often accompanied by significant symptoms, functional impairment, and caregiving burden, can be addressed as early as possible. Survivorship with stroke differs because of the acute onset of disability and relatively short time of treatment, and frequent communication impairments that complicate symptom assessment and end-of-life decision-making. The issue of living long-term with severe impairments is important in decision-making, especially when considering life-sustaining treatments such as tube feeding despite uncertain prognosis for functional recovery.

The role of rehabilitation in stroke survivorship is critical for optimizing functional outcomes and symptomatic relief, particularly in more severe strokes. Like palliative care, rehabilitation services are best incorporated across the continuum of care, starting in the acute post-stroke period as soon as the patient is medically stable. Physical and occupational therapists and speech and language pathologists should be consulted, as well as rehabilitation physicians (Table 1). Care should focus on assessment, education, and therapeutic interventions to optimize positioning and to initiate mobilization; activities of daily living (ADL) training; assessment of tone, swallowing, communication, and cognition; and orthotic evaluation.

Post-acute care rehabilitation options depend on the severity of impairments and therapy tolerance. Those with mild strokes are usually appropriate for home discharge with home or outpatient therapies, while those with more profound deficits are better served with an inpatient rehabilitation stay, either subacute (typically 1.5 hours of therapy comprised of one or more therapy disciplines, provided in a nursing home setting) or acute (3 hours of therapy and two therapy disciplines). In the acute level of care, rehabilitation physician services are rendered daily. 
Table 1. Specialty Services for Palliative Care and Rehabilitation in Stroke

\begin{tabular}{|c|c|}
\hline Specialty Service & Key Function(s) \\
\hline \multicolumn{2}{|l|}{ Palliative Care } \\
\hline $\begin{array}{l}\text { Team may include physicians, advanced } \\
\text { practice nurses, pharmacists, social } \\
\text { workers, and/or chaplains }\end{array}$ & $\begin{array}{l}\text { Multidisciplinary care for symptom management, psychosocial and spiritual support, care } \\
\text { planning and coordination and communication, including end-of-life decision-making. }\end{array}$ \\
\hline \multicolumn{2}{|l|}{ Rehabilitation } \\
\hline Physical therapy & $\begin{array}{l}\text { Assessment and rehabilitation focusing predominantly on lower extremity, gait, transfers, and } \\
\text { balance. Use physical modalities, assistive devices, and orthotics to improve function. }\end{array}$ \\
\hline Occupational therapy & $\begin{array}{l}\text { Assessment and rehabilitation focusing on ADLs, IADLs, and coordination. Incorporate } \\
\text { orthotics and adaptive equipment to maximize functioning. }\end{array}$ \\
\hline Speech and Language Pathology & Assessment and rehabilitation focusing on swallowing, communication, and cognition. \\
\hline $\begin{array}{l}\text { Physical Medicine and Rehabilitation } \\
\text { Physician or Physiatrist }\end{array}$ & $\begin{array}{l}\text { Physician specializing in assessing relationships among disease, impairments, activity and } \\
\text { participation; able to optimize medical management to enhance participation in the } \\
\text { rehabilitation program; also determine functional prognosis. }\end{array}$ \\
\hline Vocational Therapy & Provide counseling regarding employment options and adaptations to optimize job performance. \\
\hline Prosthetists/Orthotists & $\begin{array}{l}\text { Orthotists fabricate braces, and prosthetists create artificial limbs. These practitioners usually } \\
\text { have training in both disciplines. }\end{array}$ \\
\hline Recreational Therapy & $\begin{array}{l}\text { Provide therapy in inpatient rehabilitation settings which focuses on using leisure skills to } \\
\text { promote functional gains and educate patients about recreational options. }\end{array}$ \\
\hline $\begin{array}{l}\text { Rehabilitation Psychology and } \\
\text { Neuropsychology }\end{array}$ & $\begin{array}{l}\text { Rehabilitation psychologists perform evaluations and interventions to facilitate coping and } \\
\text { adaptation to disability } \\
\text { Neuropyschologists evaluate and manage behavioral and emotional issues and perform } \\
\text { cognitive testing and cognitive intervention. }\end{array}$ \\
\hline
\end{tabular}

Many acute rehabilitation facilities offer specialized stroke rehabilitation services. The goal is to provide comprehensive care which is patient-centered. Like palliative care, rehabilitation is based on a team approach, comprised of nurses typically with rehabilitation nursing specialty certification, pharmacists, nutritionists, rehabilitation and/or neuropsychologists, physiatrists, discharge planners, social workers, physical therapists, occupational therapists, speech and language pathologists, and/or recreational therapists. Rehabilitation psychologists and neuropsychologists may assist patients with coping, mood, and adjustment to medical illness and associated impairments and can perform cognitive assessments. These services are beneficial in both the inpatient and outpatient setting. Palliative care services should be considered as a part of the rehabilitation team, particularly with the more severe stroke population, and may be helpful in care planning and providing additional symptom support and spiritual care to help address issues of meaning, purpose, connection, and need for support.

Creutzfelt et $\mathrm{al}^{1}$ describe several of the most frequent and incapacitating stroke sequelae, including central post-stroke pain, spasticity, and hemiplegic shoulder pain. The extent of impairment and onset is variable and the pain often extremely severe, interfering with rehabilitation participation. In more involved hemiplegic shoulder pain syndromes, a shoulder-hand syndrome may develop with a constellation of symptoms such as those seen in complex regional pain syndrome, and treatment is more challenging. Management should be multimodal with pharmacologic and nonpharmacologic interventions, including desensitization to enable the limb to tolerate perceived noxious stimuli and range of motion, and modalities including heat, cold, electrotherapy, and massage. If pain is not sufficiently alleviated with these therapies, sympathetic blocks can be attempted and if all else fails, sympathectomies and spinal cord stimulators are possibilities. $^{3}$ A recent systematic review ${ }^{4}$ of randomized trials of techniques for management of hemiplegic shoulder pain found only eight trials, many with limited sample size. Evidence supports the use of aromatherapy with acupressure, massage, and intramuscular neuromuscular electrical stimulation; overall, Botulinum toxin A injection or intraarticular steroid injection did not show evidence of effect.

As in management of other pain conditions, psychological services consisting of pain behavior strategies and coping should be considered, particularly for central poststroke pain. Other newer therapies for pain such as motor cortex stimulation and transcranial magnetic stimulation are emerging with some success, particularly in refractory cases. Spasticity can also be a major impediment to function of the involved limb. Once exacerbating factors are eliminated such as infection, venous thromboembolism, or pain, then treatment with positioning, bracing, medications, functional electrical stimulation (stimulation to achieve a desired functional outcome such as walking), range of motion, and therapeutic interventions for motor recovery should be implemented. Medications to treat spasticity should be utilized judiciously as they can cause sedation and weakness due to relaxation in unaffected body parts. For spasticity restricted to one or two limbs or in those who cannot tolerate antispasticity medications, botulinum toxin or phenol injections may be effective. ${ }^{3}$

Addressing cognitive, visual, and bowel and bladder issues are also critical, and can benefit from a holistic, teambased approach. For visual impairment, referral for neuroophthalmology evaluation and occupational and low vision therapy services may be beneficial. Caregiving and medication administration guidance with nurses, practical assis- 
tance and helping with family dynamics with social workers, and integrating psychological, psychiatric, and spiritual care may all be valuable in severely affected patients.

Correctly diagnosing and treating clinically significant anxiety and depression, while adequately addressing other common issues such as demoralization, grief, social role functioning, and family conflict, ideally requires skilled psychosocial assessment and use of counseling and other non-medical resources. Spiritual distress can encompass much more than fears about dying: issues of meaning, purpose, and connection, including changes and guilt for patients who can no longer assume the same role through work or within the family, can all be part of the spiritual domain and are addressable through these types of interventions. The need to reframe hope and help promote acceptance and adjustment may be particularly relevant to patients whose functional and/or cognitive limitations require reassessment and reorientation of daily living and future goals. Finally, the focus of both the palliative care and rehabilitation approaches on the patient and family as a unit is particularly relevant to patients now more dependent on family members, and to family experiencing shifts in their own roles as well as caregiving burdens. ${ }^{5}$

Advances in stroke care are leading to greater survival beyond the acute and subacute stages. However, such achievements create new challenges for understanding and managing survivorship among these individuals. The holistic, interdisciplinary team approaches of both palliative care and rehabilitation services are fundamental to optimizing patient outcomes. Intervening early and helping patients and their caregivers to accept and manage associated impairments promotes more favorable results. Increased awareness about post-stroke impairments and symptoms and their treatments can facilitate timely palliative and rehabilitation interventions to improve overall survivorship and quality of life.

Corresponding Author: Sydney M. Dy, MD, MSc; Room 609, 624 N Broadway, Baltimore, MD 21205, USA (e-mail: sdy@jhsph.edu).

\section{REFERENCES}

1. Creutzfeldt CJ, Holloway RG, Walker M. Symptomatic and Palliative Care for Stroke Survivors. J Gen Intern Med. 2012. doi:10.1007/s11606011-1966-4.

2. Wee B, Adams A, Eva G. Palliative and end-of-life care for people with stroke. Curr Opin Support Palliat Care. 2010;4:229-232.

3. Gonzalez-Fernandez M, Feldman D. Stroke Rehabilitation. In: Bope ET, Kellerman RD, eds. Conn's Current Therapy 2012. Philadelphia: Elsevier; 2011.

4. Koog YH, Jin SS, Yoon K, Min BI. Interventions for hemiplegic shoulder pain: systematic review of randomised controlled trials. Disabil Rehabil. 2010;32:282-291.

5. National Consensus Project for Quality Palliative Care. Clinical Practice Guidelines for Quality Palliative Care, Second Edition, 2009. http:// www.nationalconsensusproject.org. Retrieved February 20, 2012. 